\title{
Spectrally-Efficient WDM Nyquist Pulse-Shaped Subcarrier Modulation Using a Dual-Drive Mach-Zehnder Modulator and Direct Detection
}

\author{
M. Sezer Erkılınç, Student Member, IEEE, Manoj P. Thakur, Member, IEEE, Stephan Pachnicke, Senior \\ Member, IEEE, Helmut Griesser, Member, IEEE, John Mitchell, Senior Member, IEEE, \\ Benn C. Thomsen, Member, IEEE, Polina Bayvel, Fellow, IEEE, and Robert I. Killey, Member, IEEE
}

\begin{abstract}
High data transmission capacity is increasingly needed in short- and medium-haul optical communication links. Cost-effective wavelength division multiplexed (WDM) transceiver architectures, achieving high information spectral densities (ISDs) $(>1 \mathrm{~b} / \mathrm{s} / \mathrm{Hz})$ and using low-complexity direct detection receivers are attractive solutions for such links. In this paper, we assess the use of dual-drive Mach-Zehnder modulators (DD-MZMs), and compare them with in-phase quadrature (IQ)modulators for generating spectrally-efficient single sideband Nyquist pulse-shaped 16-QAM subcarrier (N-SCM) modulation format signals. The impact of the extinction ratio (ER) of a modulator on the optical sideband suppression ratio (OSSR) was investigated for the SSB signals in WDM systems, together with the resulting impact on inter-channel crosstalk penalties. First, in back-to-back operation, an IQ-modulator with an ER of 30 $\mathrm{dB}$ and a DD-MZM with an ER of $18 \mathrm{~dB}$ were experimentally compared in a $6 \times 25 \mathrm{~Gb} / \mathrm{s}$ WDM system by varying the channel spacing. Following this comparison, $16 \mathrm{GHz}$-spaced $6 \times 25 \mathrm{~Gb} / \mathrm{s}$ WDM signal transmission was experimentally demonstrated using the DD-MZM. The experiment was performed using a recirculating loop with uncompensated standard single-mode fiber (SSMF) and EDFA-only amplification. The maximum achievable transmission distances for single channel and WDM signals were found to be 565 and $242 \mathrm{~km}$, respectively, at a net optical ISD of $1.5 \mathrm{~b} / \mathrm{s} / \mathrm{Hz}$. This is the first experimental comparison of modulator types for SSB N-SCM signal generation and the highest achieved ISD using a DD-MZM in direct-detection WDM transmission.
\end{abstract}

Index Terms-Optical fiber communication, dual-drive MZM, direct detection, WDM, ISD, subcarrier modulation, Nyquist pulse shaping, SSB, electronic pre-distortion.

\section{INTRODUCTION}

Manuscript received May 22, 2015. This work was supported by the EU ERA-NET+ project PIANO+ IMPACT, EPSRC UNLOC EP/J017582/1 and EU FP7 project ASTRON.

M. Sezer Erkılınç, Benn C. Thomsen, Polina Bayvel and Robert I. Killey are with the Optical Networks Group, Department of Electronic and Electrical Engineering at University College London, London WC1E 7JE, U.K. (e-mail: m.erkilinc@ee.ucl.ac.uk; b.thomsen@ucl.ac.uk; p.bayvel@ucl.ac.uk; r.killey@ucl.ac.uk).

Manoj P. Thakur and John Mitchell are with the Communications and Information Systems Group, Department of Electronic and Electrical Engineering at University College London, London WC1E 7JE, U.K. (email:manoj.thakur@ucl.ac.uk; j.mitchell@ucl.ac.uk).

S. Pachnicke is with the ADVA Optical Networking SE, Maerzenquelle 1-3, 98617 Meiningen, Germany. (spachnicke@advaoptical.com).

H. Griesser is with the ADVA Optical Networking SE, Fraunhoferstr. 9a, 82152 Martinsried, Germany. (hgriesser@advaoptical.com).

Color versions of one or more of these figures in this paper are available online at http://www.ieeexplore.iee.org.
Q pectrally-efficient modulation schemes achieving infor$\checkmark$ mation spectral densities (ISDs) greater than $1 \mathrm{~b} / \mathrm{s} / \mathrm{Hz}$ using direct detection receivers are attractive for access, metropolitan, and regional links due to their simplicity and low-cost. In such links, the cost-effectiveness is the primary requirement coupled with low power consumption. To achieve this, the optical complexity of the transceiver architecture needs to be minimized using low-cost and low-complexity optical components. Although the highest channel bit rates and ISDs are achievable using coherent receivers with polarization multiplexing [1] [3], direct detection receiver-based solutions, i.e., using a single-ended photodiode with no delay interferometer(s) and a single analogue-to-digital converter (ADC), are potentially preferable for metro networks since such receivers significantly reduce the costs. Network operators are installing $100 \mathrm{~Gb} / \mathrm{s}$ solutions, comprising four dense wavelength division multiplexed (DWDM) channels carrying $28 \mathrm{~Gb} / \mathrm{s}$ per wavelength, to support data transmission over metro distances [4].

To achieve ISDs greater than $1 \mathrm{~b} / \mathrm{s} / \mathrm{Hz}$ at low-cost, multilevel modulation schemes, such as 16-ary quadrature amplitude modulation (16-QAM) or higher, and electronic equalization can be implemented through the use of digital signal processing (DSP)-based transceivers. It is expected that the use of high sampling rate digital-to-analogue converters (DACs)\&ADCs will be acceptable in future low-cost systems, as the performance of silicon complementary metal oxide semiconductor technology continues to increase, whereas the cost and power consumption reduce. Therefore, to reduce the cost, each transmitter should employ a simple modulator with a high linewidth laser (typically $>200 \mathrm{kHz}$ ) and a simple receiver consisting of a single-ended photodiode. Amongst the formats offering high ISD $(>1 \mathrm{~b} / \mathrm{s} / \mathrm{Hz})$ and good dispersion tolerance $(>100 \mathrm{~km})$, dispersion pre-compensated single sideband (SSB) Nyquist pulse-shaped subcarrier modulation (N-SCM) has been shown to be one of the strongest candidates [5]-[10].

An optical single sideband signal can be generated using either an intensity modulator combined with an optical sideband filter [11] or a dual input optical modulator, such as an in-phase quadrature (IQ)-modulator [7]-[10], [12] or a dualdrive Mach-Zehnder modulator (DD-MZM) [13]-[16]. If one of the sidebands is partially suppressed, it is referred to as vestigial sideband (VSB) signalling [17]. Compared to the IQ- 
modulator, the DD-MZM has a simpler structure and a smaller footprint, and offers lower optical loss.

In the work described in this paper, a performance comparison between an IQ-modulator and a DD-MZM in a spectrallyefficient WDM system was carried out. The system operated at a net bit rate of $24 \mathrm{~Gb} / \mathrm{s}$ per channel (a gross bit rate of $25 \mathrm{~Gb} / \mathrm{s}$ ), taking into account the hard-decision forward error correction (HD-FEC) overhead. The impact of the extinction ratio on the optical sideband suppression ratio, that affects the inter-channel crosstalk, limiting the channel spacing, and consequently, the achievable spectral efficiency, was assessed in simulations and experimentally. The WDM channel spacing was varied from 12 to $20 \mathrm{GHz}$ (net optical ISDs from 2 to $1.2 \mathrm{~b} / \mathrm{s} / \mathrm{Hz}$ ) for the extinction ratio values of $10-40 \mathrm{~dB}$. Note that such (non-conventional) channel spacing values are chosen in order to maximize the ISD with the available DACs, RF-amplifiers and modulators.

Following the back-to-back comparison, a study on the use of DD-MZMs for spectrally-efficient WDM (16 GHzspaced $6 \times 25 \mathrm{~Gb} / \mathrm{s}$ ) SSB Nyquist pulse-shaped 16-QAM SCM signal transmission, yielding a net optical ISD of $1.5 \mathrm{~b} / \mathrm{s} / \mathrm{Hz}$, was carried out. The chromatic dispersion accumulated along the fiber link was compensated using electronic pre-distortion (EPD) [18]-[20]. All the channels were successfully transmitted over $242 \mathrm{~km}$ of uncompensated standard single-mode fiber (SSMF), achieving a bit-error ratio (BER) of below $3.8 \times 10^{-3}$, taken as the HD-FEC threshold.

\section{Review of Optical SSB Signal Generation USING IQ-MODULATORS AND DD-MZMS}

In this section, the principle of operation of the IQ-modulator and DD-MZM are briefly discussed. We highlight the impact of the extinction ratio (ER) of the modulator on the optical sideband suppression ratio (OSSR) in the generation of optical SSB signals in DWDM systems at ISDs greater than $1 \mathrm{~b} / \mathrm{s} / \mathrm{Hz}$.

A DD-MZM, sometimes referred to as dual-electrode MZM (DE-MZM) or polar modulator, consists of two phase modulators, driven by two independent electrical signals $\left(V_{1}\right.$ and $\left.V_{2}\right)$ [21]. The input optical field $\left(E_{\text {in }}\right)$ is split into two arms and phase modulation is applied to both arms. Then, the phasemodulated optical fields are coupled to generate the output optical field $\left(E_{\text {out }}\right)$. If the insertion loss of the MZM is ignored, the DD-MZM input-output relationship is given by

$E_{\text {out }}=E_{\text {in }}\left[\gamma \exp \left(j \pi \frac{\left(V_{1}+V_{d c}\right)}{V_{\pi}}\right)+(1-\gamma) \exp \left(j \pi \frac{V_{2}}{V_{\pi}}\right)\right]$,

where $\gamma$ is the optical splitting ratio with a value between 0 and $1 . V_{\pi}$ is the differential voltage between the two electrodes to provide a $\pi$ phase-shift between the two waveguides, and $V_{d c}$ is the applied DC bias voltage. Note that the terminal for $V_{2}$ is assumed to be grounded. Having two independent phase modulators enables to generate two phase-modulated signals. These signals can be used to implement arbitrary amplitude and phase modulation, such as high-order QAM signalling. However, the driving signals $\left(V_{1}\right.$ and $\left.V_{2}\right)$ need to be correctly mapped from Cartesian to polar coordinates as described in [22], [23].
An IQ-modulator consists of two intensity modulators (typically two single-drive MZMs) and a phase shifter. The input optical field is split into two paths, the I and Q arms. The optical field amplitude modulation is achieved by biasing the single-drive MZMs at their minimum transmission (null) point $\left(V_{I d c}=V_{Q d c}=0\right)$. With a relative $\pi / 2$ phase-difference induced by the phase modulator, the output optical field can realize any constellation point(s) on the complex IQ-plane (in Cartesian coordinates) after the outputs of the two arms are coupled. The output optical field can be defined as

$E_{\text {out }}=E_{\text {in }}\left[\gamma \cos \left(\frac{V_{I}+V_{I d c}}{V_{\pi}} \pi\right)+j(1-\gamma) \cos \left(\frac{V_{Q}+V_{Q d c}}{V_{\pi}} \pi\right)\right]$,

where $V_{I}$ and $V_{Q}$ are the electrical driving signals.

To generate an optical SSB signal using a dual-input optical (vector) modulator, such as a DD-MZM or an IQ-modulator, the two independent arms need to be driven such that the unwanted sideband from each arm of the modulator interfere destructively, while the desired ones interfere constructively. Therefore, the time dependent driving signals, $V_{1}$ and $V_{2}$, are

$$
V_{1}=\frac{V_{1_{r m s}}}{V_{\pi}}\left[x_{r}(t)-\hat{x}_{i}(t)\right] \quad \text { and } \quad V_{2}=\frac{V_{2_{r m s}}}{V_{\pi}}\left[\hat{x}_{r}(t)+x_{i}(t)\right] \text {, }
$$

where $x_{r}(t)$ and $x_{i}(t)$ are the real and imaginary parts of a time dependent signal, $\hat{x}_{r}(t)$ and $\hat{x}_{i}(t)$ are their Hilbert transforms, and $V_{1_{r m s}}$ and $V_{2_{r m s}}$ are the root mean square value of the driving signals, respectively. The Hilbert transform is a process in which all negative frequency components of a signal are phase-advanced by $90^{\circ}$ whereas all positive frequency components are phase-delayed by $90^{\circ}$. The amplitude of the spectrum remains unchanged. In other words, it introduces a $180^{\circ}$ phase difference between the negative and positive frequency components of an input signal [24]-[26]. If the electrical signal $x(t)$ is real-valued, $x_{i}(t)$ and $\hat{x}_{i}(t)$ become zero. Otherwise, a butterfly structure needs to be used, e.g., if the signal is pre-distorted/dispersed to mitigate the chromatic dispersion. In the case of the DD-MZM, the signal waveforms generated in Cartesian coordinates need to be converted to polar coordinates using Eq.6 and Eq.7 in [22]. Note that in all cases, the attenuations and the phase of the driving signals should be controlled identically to obtain a maximum sideband suppression.

The ER of an optical modulator is crucial in the generation of an optical SSB signal. If the input optical field is not equally split, the unwanted sideband is not fully suppressed, causing a spectral broadening. Hence, linear crosstalk between the neighbouring WDM channels is observed (assuming no optical filtering is used when the WDM channels are combined, e.g., if it is carried out with an optical coupler), and consequently, it causes penalties at channel spacing values of less than twice the SSB signal bandwidth. The ER of an optical modulator, which is related to $\gamma$, is given by

$$
\mathrm{ER}(\mathrm{dB})=-20 \log _{10}(2 \gamma-1) \approx \operatorname{OSSR}(\mathrm{dB}) .
$$

Moreover, if the SSB signal is generated utilizing the Hilbert transform, the optical sideband suppression (OSSR), defined as the power of the desired sideband divided by the power of 
the suppressed sideband, is approximately equal to the ER, assuming the attenuation and the phase on both arms are optimized. The change in OSSR and ER with respect to $\gamma$ are shown in Fig. 1 along with the simulated optical spectra at certain ER values. For instance, if the incoming light is split with a ratio of $0.55\left(0.55 E_{\text {in }}\right.$ to one arm and $0.45 E_{\text {in }}$ to the other arm), the ER of the modulator is $20 \mathrm{~dB}$, meaning that the unwanted sideband can be suppressed by up to approximately $20 \mathrm{~dB}$, as can be seen in Fig. 1 .
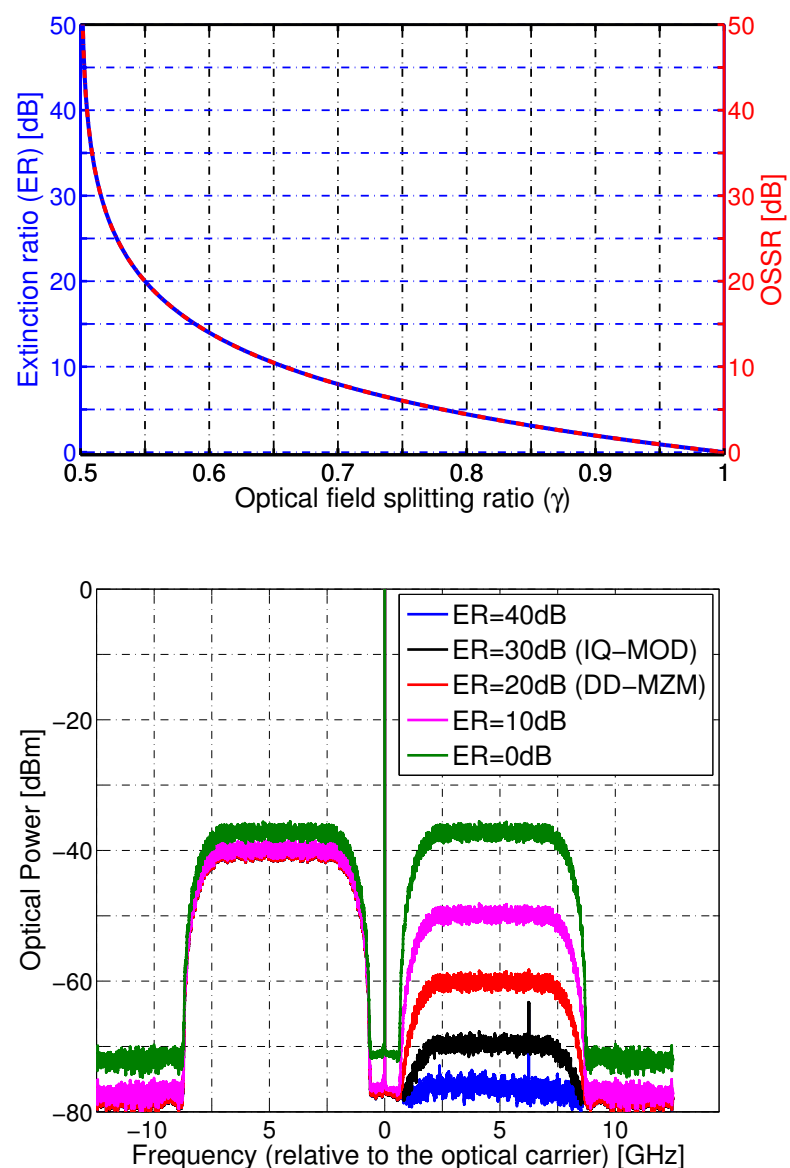

Fig. 1: ER and OSSR with respect to $\gamma$ of the optical modulator (top). Simulated optical spectra at a resolution of $10 \mathrm{MHz}$ for different extinction ratio values (bottom).

\section{Numerical Simulations ANd ExPERIMENTAL SETUP}

In this section, the simulation model for the Nyquist pulseshaped SCM signal generation, transmission and detection along with the (offline) experimental waveform generation is described in detail. Then, the experimental setup is outlined.

\section{A. Numerical Simulations and Offline Waveform Generation}

The simulation model for the Nyquist pulse-shaped 16-QAM SCM signal generation, transmission and detection was implemented in MATLAB. Four $2^{18}$ patterns, based on de Bruijn bit sequences and decorrelated by 0.25 of the pattern length, were mapped to 16-QAM symbols at a symbol rate of $f_{s}=6.25$ GBaud. After bit-to-symbol mapping, a pair of

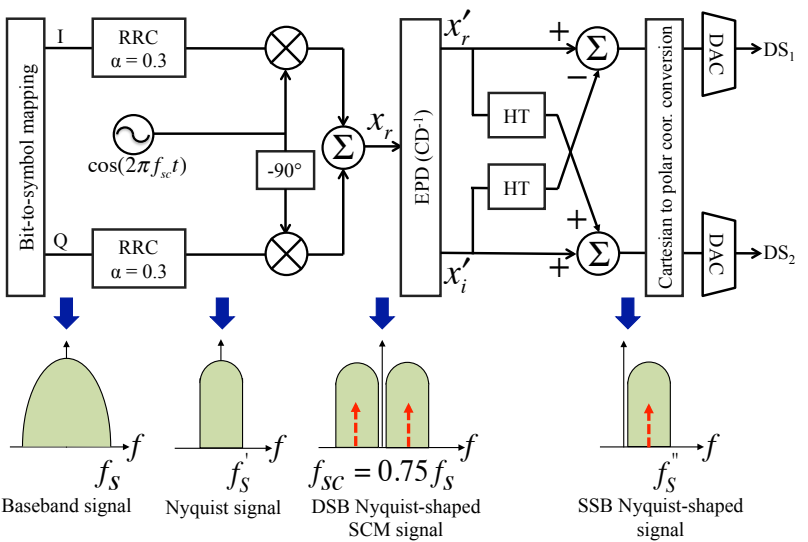

Fig. 2: Block diagram of Nyquist-SCM Transmitter DSP (top) and the schematic of the signal spectra of DSB and SSB three quartercycle $\left(f_{s c}=0.75 f_{s}\right)$ Nyquist pulse-shaped ( $\left.\alpha=0.3\right)$ SCM (bottom). $x_{r}$ : real-valued DSB Nyquist-SCM signal. $\hat{x}_{r}(t)$ and $\hat{x}_{i}(t)$ : real and imaginary parts of the pre-dispersed SSB Nyquist-SCM signal. $f_{s}$ : symbol rate, $f_{s c}$ : subcarrier frequency, $f_{s}^{\prime}=f_{s} / 2(1+\alpha), f_{s}^{\prime \prime}=f_{s c}+f_{s}^{\prime}$, EPD: Electronic pre-distortion and CD: Chromatic dispersion.

root raised-cosine (RRC) pulse-shaping filters with a roll-off factor $(\alpha=)$ of $0.3,256$ taps and a stop-band attenuation of $40 \mathrm{~dB}$ were applied to the I- and Q-baseband signals. The baseband signals were up-converted to a subcarrier frequency $\left(f_{s c}\right)$ of $4.68 \mathrm{GHz}\left(0.75 \times f_{s}\right)$ and added to each other to generate a real-valued DSB Nyquist pulse-shaped SCM signal, denoted as $x_{r}(t)$, at a bit rate of $25 \mathrm{~Gb} / \mathrm{s}$, as shown in Fig. 2. Dispersion pre-compensation was implemented by applying the inverse of the transfer function of the fiber (neglecting loss and nonlinearity) to mitigate the dispersion, as described in [18]-[20]. Finally, two 6-bit quantized pre-dispersed signals, $\mathrm{DS}_{1}=x_{r}(t)-\hat{x}_{i}(t)$ and $\mathrm{DS}_{2}=\hat{x}_{r}(t)+x_{i}(t)$, were used to achieve SSB signalling, as shown in Fig. 2. Cartesian to polar coordinate conversion was performed when using a DD-MZM, as given by Eq.6 and Eq.7 in [22].

The effective number of bits (ENOB) of the DACs used in the experiment was measured to be 3.8 bits at $10 \mathrm{GHz}$. Therefore, in the simulations, the electrical signal-to-noise ratio (SNR) of the driving signals was set to $23 \mathrm{~dB}$ to emulate the DAC quantization noise. The low-pass filters (LPFs) used in the experiment to remove the images generated by the DACs were modeled as $5^{\text {th }}$-order Bessel filters with a bandwidth of $7 \mathrm{GHz}$. The single channel SSB signal was generated using Eq. 1 for the DD-MZM with an ER of $18 \mathrm{~dB}(\gamma=0.56)$ and Eq.2 for the IQ-modulator with an ER of $30 \mathrm{~dB}(\gamma=0.51)$, respectively. As the optical source, a distributed feedback (DFB) laser with a linewidth of $1 \mathrm{MHz}$ was used, operating at $1550 \mathrm{~nm}$. In WDM simulations, the channels carrying 25 Gb/s SSB Nyquist pulse-shaped 16-QAM SCM signal were decorrelated by approximately 1000 symbols.

The symmetric split-step Fourier method [27] was utilized to model the single channel and WDM signal transmission at a simulation bandwidth of $200 \mathrm{GHz}$ with step sizes of $1 \mathrm{~km}$ and $400 \mathrm{~m}$, respectively. The fiber parameters $\left(\alpha, D, \gamma\right.$ and $\left.L_{\text {span }}\right)$ were $0.2 \mathrm{~dB} / \mathrm{km}, 17 \mathrm{ps} /(\mathrm{nm} . \mathrm{km}), 1.2 \mathrm{~W}^{-1} \mathrm{~km}^{-1}$ and $80 \mathrm{~km}$, respectively. To emulate the nonlinear interaction between the signal and amplified spontaneous emission (ASE)-noise, all ASE-noise generated by the EDFAs was added inline. 


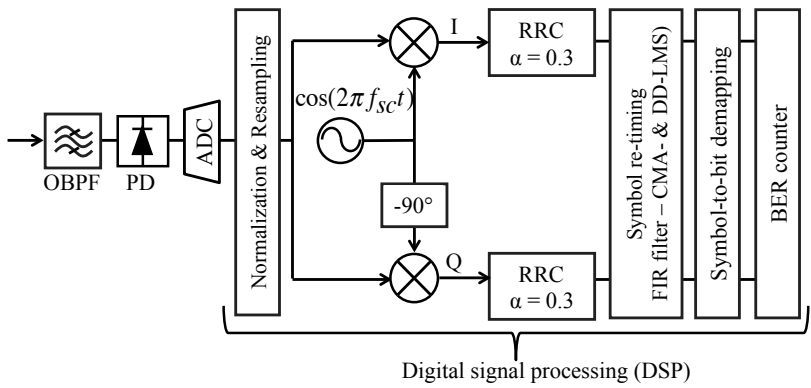

Fig. 3: Block diagram of Nyquist-SCM receiver. CMA: Constant modulus algorithm, DD: Decision-directed, LMS: Least mean squares and FIR: Finite-impulse response.

Before photodetection using a single-ended photodiode with a responsivity of $0.8 \mathrm{~A} / \mathrm{W}$, a $4^{\text {th }}$-order super-Gaussian optical band-pass filter (OBPF) was applied to demultiplex the channel of interest and remove out-of-band ASE-noise. Following the resampling and quantization of the digitized signal by a single ADC with an ENOB of 5-bit at $10 \mathrm{GHz}$ and a sampling rate of $50 \mathrm{GSa} / \mathrm{s}$, a $5^{\text {th }}$-order Bessel LPF with a bandwidth of $16 \mathrm{GHz}$ was used to emulate the frequency response of the real-time sampling scope used in the experiment. The block diagram of the receiver DSP is shown in Fig. 3. The resampled quantized signal was first split into two branches, and subsequently, down-converted to generate the I- and Qbaseband signals. A pair of matched RRC filters with $\alpha=$ 0.3 were used, followed by a 5-tap FIR filter for symbol retiming and the BER counter. Initially, the constant modulus algorithm was chosen as a cost function for fast convergence, and then, switched to decision directed least-mean squares (LMS). Finally, the BER was computed by error counting over $2^{20}$ bits. To calculate the upper bounds on the net bit rate and net optical ISD, the hard decision decoding bound for the binary symmetric channel was utilized resulting in a maximum code rate $(r)$ of [28]

$$
r=1+p_{b} \log _{2} p_{b}+\left(1-p_{b}\right) \log _{2}\left(1-p_{b}\right),
$$

where $p_{b}$ is the BER. Using Eq.5, $r$ was found to be 0.96 at a $p_{b}$ of $3.8 \times 10^{-3}$, yielding a net bit rate of $24 \mathrm{~Gb} / \mathrm{s}$ per channel (a gross bit rate of $25 \mathrm{~Gb} / \mathrm{s}$ ) and a net optical ISD of $1.5 \mathrm{~b} / \mathrm{s} / \mathrm{Hz}$ (a gross optical ISD of $1.56 \mathrm{~Gb} / \mathrm{s}$ ) in the WDM transmission. The simulation and experimental results are discussed together in Section IV.

\section{B. Experimental Setup}

The optical transmission test-bed used for the experiment consisted of a WDM SSB Nyquist pulse-shaped 16-QAM subcarrier modulation transmitter, an optical fiber recirculating loop and a direct detection receiver, as shown in Fig. 4. As previously described in Section III-A, the driving signal waveforms for the modulators, ' $\mathrm{DS}_{1}$ ' and ' $\mathrm{DS}_{2}$ ', were generated offline in MATLAB using $2^{15}$ de Bruijn bit sequences, as shown in Fig. 2. The waveforms were quantized to 6 bits for the DACs (Micram VEGA DACII) with an ENOB of 3.8 bits at $10 \mathrm{GHz}$ operating at a sampling rate of $25 \mathrm{GSa} / \mathrm{s}$, and uploaded to the memory of a pair of Xilinx Virtex- 5 FPGAs' RAM blocks. To prevent linear crosstalk between neighbouring channels due to the images generated by the DACs, electrical anti-imaging filters, $5^{\text {th }}$-order Bessel LPFs with a bandwidth of $7 \mathrm{GHz}$, were used. The $\mathrm{LiNbO}_{3}$ IQmodulator with a $V_{\pi}$ of $3.5 \mathrm{~V}$ was driven by the electrical signals with a $V_{p p}$ of $3.4 \mathrm{~V}$ whereas the $V_{p p}$ of the driving signals was set to $2.4 \mathrm{~V}$ for the $\mathrm{LiNbO}_{3}$ DD-MZM with a $V_{\pi}$ of $2.6 \mathrm{~V}$. The optical carrier was added by biasing the modulator (IQ-modulator or DD-MZM) close to its quadrature point to achieve approximately linear mapping from the electrical to the optical domain with the bias voltages, adjusted to achieve the desired optical carrier-to-signal power ratio (CSPR). Note that the CSPR is defined as the ratio between the optical carrier and the sideband power. In the single channel case, only the DFB laser operating at $\lambda_{3}$ was used. The optical spectrum of the single channel taken from the optical spectrum analyser operating at a resolution bandwidth of $0.01 \mathrm{~nm}$ is shown in the inset (a) of Fig. 4.

In the WDM case, first, the channel spacing was varied from 12 to $20 \mathrm{GHz}$ in back-to-back operation to assess the impact of the finite optical sideband suppression on the interchannel crosstalk penalty. DFB lasers $\left(\lambda_{1,3,5}\right)$ with a linewidth of approximately $1 \mathrm{MHz}$ at $1549.60 \mathrm{~nm}$, separated by twice the channel spacing, were used as optical sources for both the IQ-modulator and DD-MZM. The odd channels $\left(\lambda_{1,3,5}\right)$ were frequency shifted by the value of the channel spacing using a separate IQ-modulator. As shown in Fig. 4, both arms of this IQ-modulator were driven by a signal generator with a tone at the frequency corresponding to the WDM channel spacing. The phase shifters on both arms were adjusted such that a $90^{\circ}$ phase difference was obtained between two arms to suppress one of the side tones by approximately $30 \mathrm{~dB}$, and the modulator was biased at its null point to suppress the light at the original frequency. The odd channels were delayed using fiber length of $3.4 \mathrm{~m}$ length (a delay of $17 \mathrm{~ns}$ corresponding to 429 samples) to achieve signal decorrelation between odd and even channels. Finally, the odd and even channels were combined using a $3 \mathrm{~dB}$ coupler to generate the WDM SSB Nyquist pulse-shaped 16-QAM SCM signal, as illustrated in Fig. 4 with its optical spectrum, given in the inset (b). The optimum channel spacing was chosen based on the trade-off between maximizing the ISD and minimizing the required OSNR penalty caused by the linear crosstalk between neighbouring channels.

Once the optimum channel spacing was determined, the transmission experiment was performed using an optical recirculating loop with a single span of $80.7 \mathrm{~km} \mathrm{SSMF}$, as shown in Fig. 4. The fiber parameters, $D, \alpha$ and $\gamma$ were $17 \mathrm{ps} / \mathrm{nm} / \mathrm{km}$, $0.2 \mathrm{~dB} / \mathrm{km}$ and $1.2 \mathrm{~W}^{-1} \mathrm{~km}^{-1}$, respectively. An OBPF (Yenista Optics XTM50-Wide) with a bandwidth of $200 \mathrm{GHz}$ and a filter edge gradient of $500 \mathrm{~dB} / \mathrm{nm}$ was used to filter the out-ofband ASE-noise during the transmission. A loop synchronous polarization scrambler (PS) was utilized to randomize the signal polarization state at each circulation. The launch power into the span was controlled by variable optical attenuators (VOAs). The fiber loss $(16 \mathrm{~dB})$ plus the insertion loss of the loop components (15 dB from VOAs, PS, AOM and OBPF) resulted in a total loss of $31 \mathrm{~dB}$ per recirculation. This loss was compensated by two EDFAs with a noise figure of $5 \mathrm{~dB}$ 


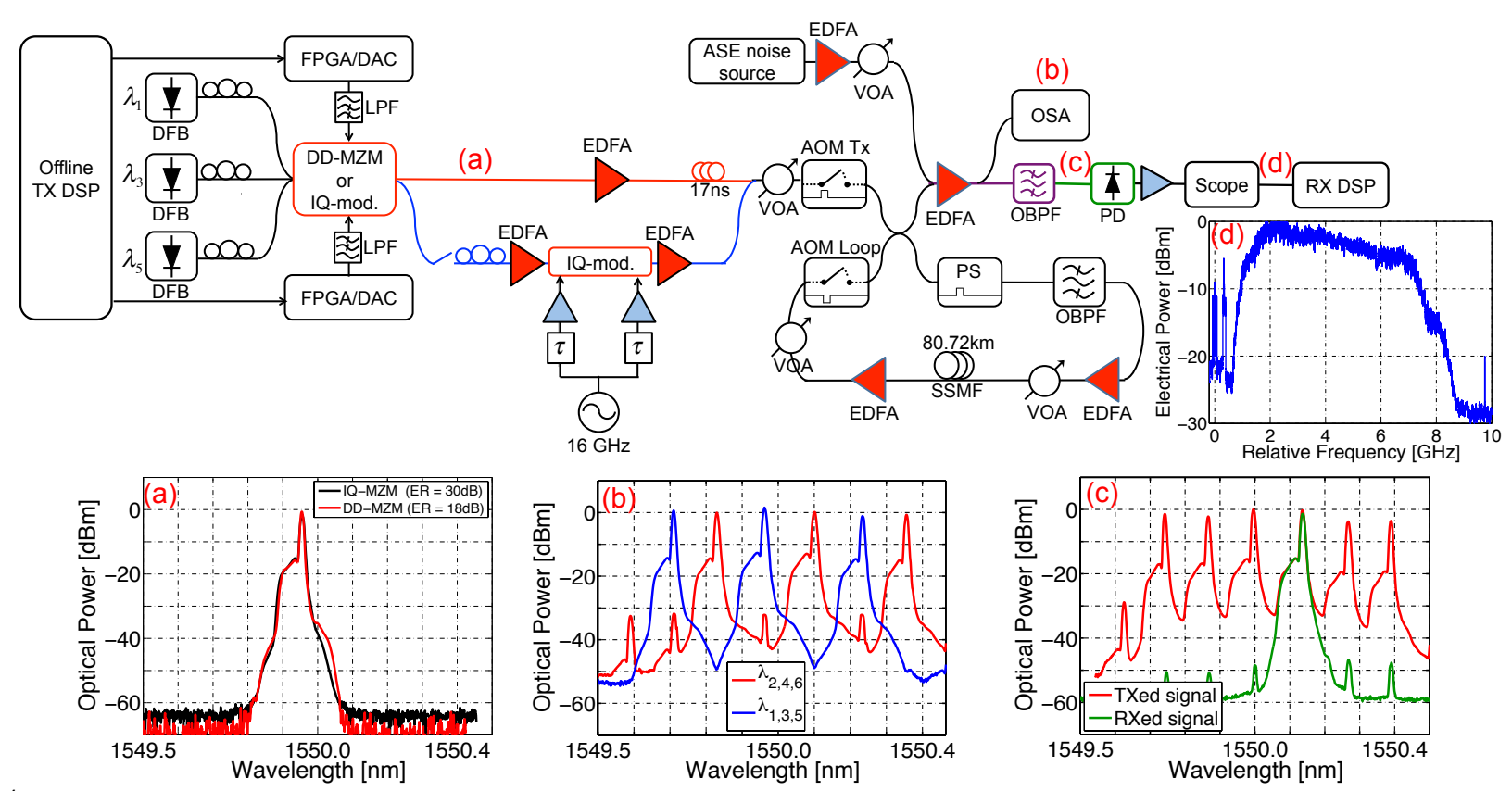

Fig. 4: Experimental setup for WDM SSB Nyquist pulse-shaped SCM transmission. FPGA: Field programmable gate array, VOA: Variable optical attenuator, AOM: Acousto-optic modulator, PS: Polarization scrambler, OBPF: Optical band-pass filter, PD: Photodiode. Insets: (a) Experimental optical spectrum of single channel generated using IQ- and DD-MZ modulators, (b) transmitted WDM signal with (c) the received optical spectrum. (d) Received electrical spectrum. Offline TX and RX DSP are shown in Fig. 2 and Fig. 3, respectively.

operating at their saturation point (18 $\mathrm{dBm}$ output power).

At the receiver, the channel of interest was demultiplexed using a manually tunable OBPF (Yenista Optics XTM50Ultrafine) with a $3 \mathrm{~dB}$ bandwidth of $2 \mathrm{GHz}$ less than the channel spacing and a filter edge gradient of $800 \mathrm{~dB} / \mathrm{nm}$, as shown in the inset (c) of Fig. 4. A single-ended PIN Discovery photodiode (DSC10H) was used to detect the filtered optical signal, followed by an RF-amplifier. The received electrical signal spectrum after digitization using a single ADC, operating at $50 \mathrm{GSa} / \mathrm{s}$ with an electrical bandwidth of $16 \mathrm{GHz}$ and a nominal resolution of 8 bits (ENOB of 5 bits at $10 \mathrm{GHz}$ ), is shown in the inset (d) of Fig. 4. The receiver DSP used in the simulations, as described in Section III-A with the block diagram presented in Fig. 3, was also used in the experiments. The BER was computed by error counting over $2^{20}$ bits.

\section{TRAnsmission Results And Discussions}

In this section, the performance of the IQ-modulator and DDMZM is compared for the single channel and WDM signals in back-to-back operation. In the WDM case, the channel spacing was varied from 12 to $20 \mathrm{GHz}$. The experimentally measured and simulated required OSNR values of the WDM SSB Nyquist pulse-shaped 16-QAM SCM signals are presented. Following the comparison, the single channel and WDM transmission results for the SSB Nyquist pulse-shaped 16QAM SCM signal generated by the DD-MZM are presented in Section IV-B.

\section{A. Back-to-back Performance}

The BER versus OSNR values for the single channel case, with the received constellations at an OSNR of $34 \mathrm{~dB}$ are shown in Fig. 5. Ideal system simulations were performed, neglecting any practical limitations such as DAC/ADC quantization noise, and non-ideal optical and electrical filtering effects. The required OSNR at the HD-FEC threshold in ideal simulations was found to be $21 \mathrm{~dB}$ at a BER of $3.8 \times 10^{-3}$ for the SSB Nyquist pulse-shaped 16-QAM SCM signal with $\alpha=0.3$ and $f_{s c}=4.68 \mathrm{GHz}\left(0.75 \times f_{s}\right)$. In our experiment, the implementation penalty, as compared to the ideal system simulations, was found to be $2 \mathrm{~dB}$ (a required OSNR of 23 $\mathrm{dB}$ ) caused by the DAC quantization noise, low-pass filtering effects and the non-ideal OBPF at the receiver before the photodiode. Moreover, there was no significant performance difference observed between the IQ-modulator and DD-MZM for the single channel case, as presented in Fig. 5.

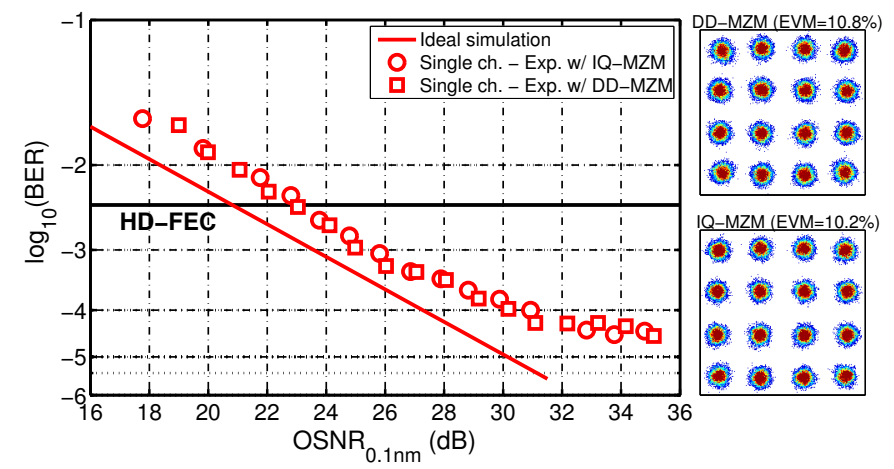

Fig. 5: Back-to-back BER vs. OSNR values for the single channel case using the IQ-modulator and DD-MZM. The received constellations at an OSNR of $34 \mathrm{~dB}$ with an EVM of $10.2 \%$ and $10.8 \%$. EVM is defined as the difference between the measured and ideal symbols, calculated as the root-mean-square (RMS) value, as given in Eq.10 in $\underline{\text { [29] }}$

After the single channel measurements, the channel spacing was varied from 12 to $20 \mathrm{GHz}$ (a net optical ISD of from 2 to $1.2 \mathrm{~b} / \mathrm{s} / \mathrm{Hz}$ ). Using the IQ-modulator and DD-MZM, the backto-back required OSNR values for the WDM system were 
compared experimentally and in practical simulations using different values of the extinction ratio (ER). The simulation setup is described in Section III-A. One of the central channels $\left(\lambda_{3}\right)$ was selected as the channel of interest during these measurements. In the practical system simulations with an ER of $40 \mathrm{~dB}$, no significant OSNR penalty was observed at channel spacing above $12 \mathrm{GHz}$. The OSNR penalty at a channel spacing of $12 \mathrm{GHz}$ is due to the non-ideal demultiplexing before the photodiode. In the experiment, using the DD-MZM, the required OSNR values were found to be 34 and $26 \mathrm{~dB}$ (the OSNR penalties of 11 and $3 \mathrm{~dB}$ compared to the single channel case) at channel spacings of 12 and $14 \mathrm{GHz}$, respectively. The values for the IQ-modulator at the same channel spacings were measured to be 24.4 and $24.1 \mathrm{~dB}$ (OSNR penalties of 1.4 and $1.1 \mathrm{~dB}$ compared to the single channel case), respectively. The OSNR penalties observed in the DD-MZM case are due to the lower suppression of the unwanted sideband compared to the IQ-modulator, resulting in higher linear crosstalk between the neighbouring channels. The suppression ratio, typically limited to $20 \mathrm{~dB}$ for a DDMZM, can be increased to $45 \mathrm{~dB}$ by cascading DD-MZM with a phase modulator, as experimentally demonstrated in [16]. At a channel spacing of $16 \mathrm{GHz}$ or more, the measured required OSNR penalties were found to be within $1 \mathrm{~dB}$ compared to the single channel performance. The experimental results for the WDM system matched well with the practical simulation results, as can be seen in Fig. 6. As a result, we chose $16 \mathrm{GHz}$ as the channel spacing value for the WDM transmission experiment using the DD-MZM, explained in Section IV-B. The optimum CSPR at the HD-FEC threshold was found to be approximately $9 \mathrm{~dB}$ for both single channel and WDM signals. The high CSPR is mainly due to the overlap between the sideband and the signal-signal beating terms, resulting in interference. In direct detection links, the optimum CSPR is dependent on the OSNR value. A detailed discussion regarding the optimum CSPR value can be found in [10]. It is worth noting that if the WDM transmission is realized using the IQ-modulator instead of DD-MZM, the channel spacing can be chosen as $12 \mathrm{GHz}$, yielding an ISD of $2 \mathrm{~b} / \mathrm{s} / \mathrm{Hz}$, as demonstrated in [10].

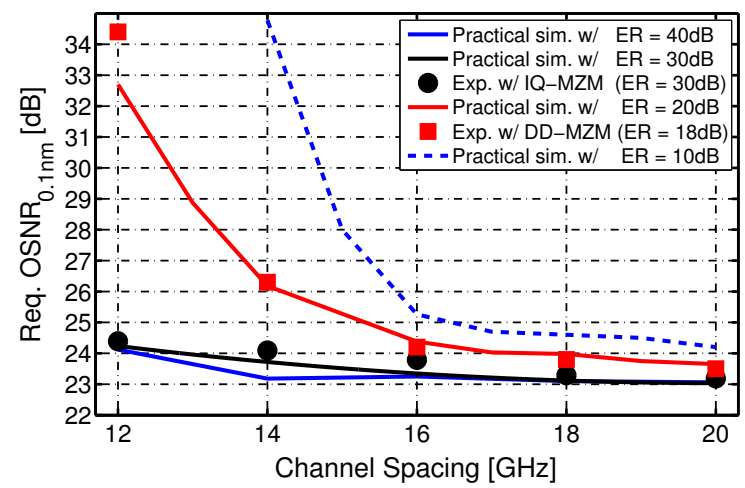

Fig. 6: Simulated and experimental required OSNR values with respect to the channel spacing using the IQ-modulator and DD-MZM.

Once the optimum channel spacing was determined using the DD-MZM, the back-to-back BER versus OSNR perfor- mance for the $16 \mathrm{GHz}$-spaced WDM $(6 \mathrm{ch} . \times 25 \mathrm{~Gb} / \mathrm{s}) \mathrm{SSB}$ Nyquist pulse-shaped 16-QAM SCM signal generated by the IQ-modulator and DD-MZM was measured, and presented in Fig. 7 along with the received constellations at an OSNR of $34 \mathrm{~dB}$. In the DD-MZM case, the BER values were measured both for the third $\left(\lambda_{3}\right)$ and fourth $\left(\lambda_{4}\right)$ channels. The frequency stability of the DFB lasers was approximately $\pm 2 \mathrm{GHz}$ which is reasonable for low-cost direct detection links over metropolitan distances. Nevertheless, this does not cause any significant penalties $(<0.5 \mathrm{~dB})$ since the signal bandwidth is $8.75 \mathrm{GHz}$ whereas the channel spacing is chosen as $16 \mathrm{GHz}$.

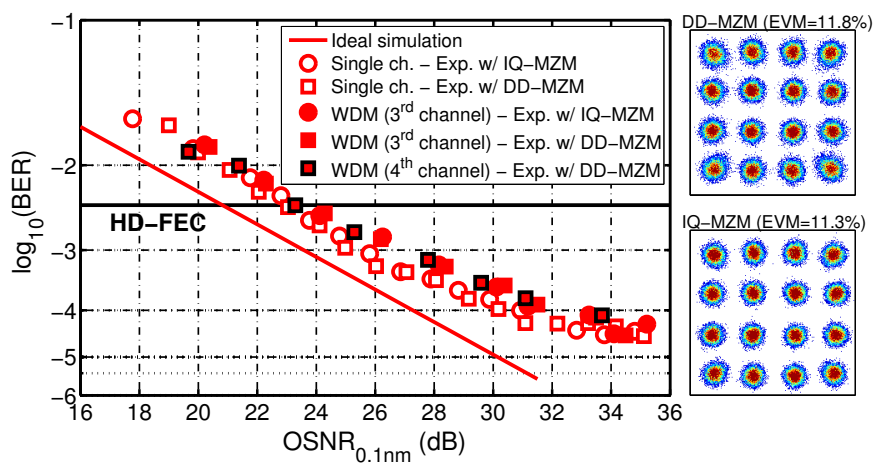

Fig. 7: BER vs. OSNR for the WDM system using the IQ-modulator and DD-MZM (left). The received constellations at an OSNR of 34 $\mathrm{dB}$ (right).

\section{B. Transmission Performance}

Following the back-to-back performance assessment, single channel and WDM signal transmission experiments were carried out using the recirculating fiber loop. The experimental measured BER values with respect to the launch power per channel, are shown in Fig. 8 including the received constellations at their optimum launch power values. The maximum achievable single channel transmission distance was $565 \mathrm{~km}$ and the optimum launch power was found to be $2 \mathrm{dBm}$, operating at an optimum CSPR of approximately $9 \mathrm{~dB}$ at the HDFEC threshold, similar to the back-to-back operation. Since there is no significant penalty observed between the single channel and WDM back-to-back performance (see Fig. 7), the transmission performancs at a given distance $(242 \mathrm{~km})$ is very similar in the linear regime, as can be observed in Fig. 8. However, the maximum achievable transmission distance was reduced from 565 to $242 \mathrm{~km}$ due to the additional inter-channel nonlinear effects during the WDM transmission. The optimum launch power per channel for the WDM signal was found to be $-2 \mathrm{dBm}, 4 \mathrm{~dB}$ less than the single channel transmission. The optimum CSPR value in WDM transmission was found to be approximately $7.5-8 \mathrm{~dB}$ at $242 \mathrm{~km}$, slightly lower that for the single channel transmission at $565 \mathrm{~km}$. This small change in the optimum CSPR value is due to the trade-off between the SSBI and fiber nonlinearities, and, as expected, the optimum CSPR value is lower in WDM transmission because of the fiber nonlinearities. The experimental BER values for WDM transmission were measured for both $\lambda_{3}$ and $\lambda_{4}$ channels after WDM transmission. 


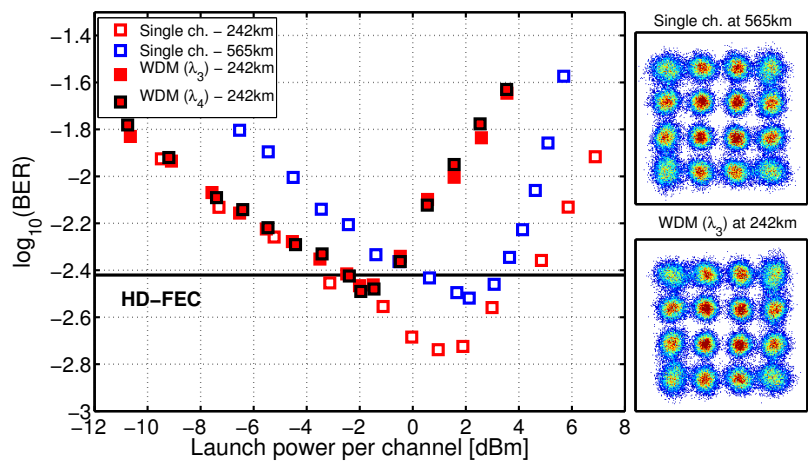

Fig. 8: BER vs. launch power per channel for WDM transmission using DD-MZM (left). The received constellations at the optimum launch power (right).

Furthermore, all six transmitted channels carrying the SSB Nyquist pulse-shaped 16-QAM SCM signal and operating at a bit rate of $25 \mathrm{~Gb} / \mathrm{s}$, generated by the DD-MZM, achieved a BER below $3.8 \times 10^{-3}$ at the optimum launch power per channel, as shown in Fig. 9 with the transmitted optical spectrum. A net optical ISD of $1.5 \mathrm{~b} / \mathrm{s} / \mathrm{Hz}$ was achieved over $242 \mathrm{~km}$ of SSMF. This is the highest achieved ISD using a DDMZM in direct detection links over this transmission distance. It is worth noting that the maximum achieved ISD in direct detection links is reported as $2 \mathrm{~b} / \mathrm{s} / \mathrm{Hz}$ at $343 \mathrm{~km}$ using an IQ-modulator [10].

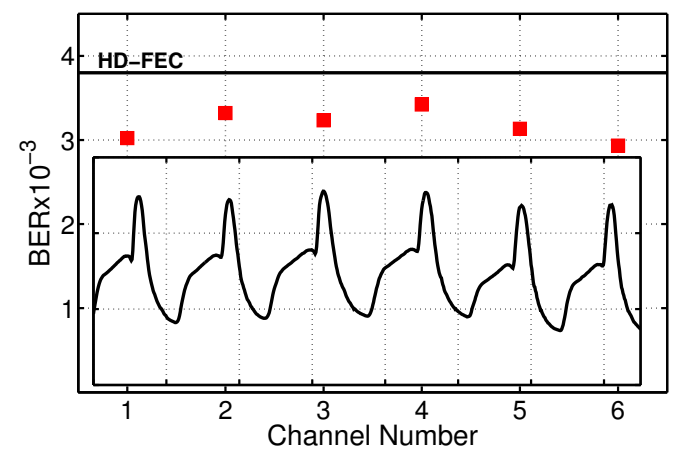

Fig. 9: The measured BER values for each received channel using DD-MZM at $242 \mathrm{~km}$. Inset: Transmitted optical spectrum.

\section{Summary AND CONCLUSIONS}

We have investigated the generation of SSB N-SCM signals using IQ- and dual-drive MZ modulators, including the effect of the finite extinction ratio of the modulator in relation to the optical sideband suppression, and the resulting crosstalk between neighbouring WDM channels. To demonstrate this relationship, we compared, both in simulations and experimentally, the back-to-back performance of $25 \mathrm{~Gb} / \mathrm{s}$ per channel WDM SSB Nyquist pulse-shaped 16-QAM SCM signaling using an IQ-modulator with an extinction ratio of $30 \mathrm{~dB}$ and a DD-MZM with an extinction ratio of $18 \mathrm{~dB}$. At a channel spacing of $16 \mathrm{GHz}$ or more, no significant differences in the required OSNR value were observed between the modulators at the HD-FEC threshold. In contrast, due to the lower optical sideband suppression, the required OSNR values increases by 2 and $10 \mathrm{~dB}$ using the DD-MZM compared to the IQmodulator at channel spacings of 14 and $12 \mathrm{GHz}$, respectively.

Following this, we carried out WDM SSB Nyquist pulseshaped 16-QAM SCM signal transmission experiments using the DD-MZM at a channel spacing of $16 \mathrm{GHz}$, achieving a net optical ISD of $1.5 \mathrm{~b} / \mathrm{s} / \mathrm{Hz}$. The required OSNR values for the single channel and WDM signals were both found to be 23.2 $\mathrm{dB}$ due to the relatively large channel spacing. The maximum achievable transmission distance over EDFA-only amplified SSMF link, with $31 \mathrm{~dB}$ loss per amplifier span, was $565 \mathrm{~km}$ for the single channel, and decreasing to $242 \mathrm{~km}$ in the WDM case due to the inter-channel nonlinear effects. Finally, it was shown that all six transmitted channels achieved BER values below the HD-FEC threshold at the maximum WDM transmission distance of $242 \mathrm{~km}$. To the best of our knowledge, this is the first experimental performance comparison of IQ-modulator and DD-MZM for SSB Nyquist pulse-shaped SCM signalling. Moreover, it is the highest achieved ISD, at this distance, among the reported experimental single polarization WDM demonstrations in direct detection links using a DD-MZM based transmitter and a direct detection receiver, comprising a single-ended photodiode with a single ADC. The experimental results indicate that the direct detection SSB Nyquist pulseshaped SCM modulation technique using compact DD-MZMs can be a promising and practical approach for metro, regional and access applications. It offers a high information spectral density with a cost-effective transceiver design.

\section{ACKNOWLEDGEMENTS}

The authors would like to thank Dr. S. Mikroulis for providing the $\mathrm{LiNbO}_{3}$ DD-MZM.

\section{REFERENCES}

[1] M. Mazurczyk, "Spectral shaping in long haul optical coherent systems with high spectral efficiency," J. Lightw. Technol., vol. 32, no. 16, pp. 2915-2924, 2014.

[2] J.X. Cai, "100G transmission over transoceanic distance with high spectral efficiency and large capacity," J. Lightw. Technol., vol. 30, no. 24, pp. 3845-3856, 2012.

[3] P. Winzer, "High spectral-efficiency optical modulation formats," $J$. Lightw. Technol., vol. 30, no. 24, pp. 3824-3835, 2012.

[4] ADVA, Efficient 100G Transport (2014). (retrieved November $1^{\text {st }} 2015$ ) http://www.advaoptical.com/en/innovation/100g-transport/100g-metro. aspx.

[5] A.S. Karar and J.C. Cartledge, " $100 \mathrm{~Gb} / \mathrm{s}$ intensity modulation and direct detection," J. Lightw. Technol., vol. 32, no. 16, pp. 2809-2814, (invited paper) 2014

[6] N. Liu, X. Chen, C. Ju, and R. Hui, "40-Gbps vestigial sideband halfcycle Nyquist subcarrier modulation transmission experiment and its comparison with orthogonal frequency division multiplexing," Opt. Eng., vol. 53, no. 9, pp. 096114-096114, 2014.

[7] M.S. Erkilinc, R. Maher, M. Paskov, S. Kilmurray, S. Pachnicke, H. Griesser, B.C. Thomsen, P. Bayvel, and R. Killey, "Spectrallyefficient single-sideband subcarrier-multiplexed quasi-Nyquist QPSK with direct detection," in Proc. ECOC, paper Tu3C4, 2013.

[8] M.S. Erkılınç, S. Kilmurray, R. Maher, M. Paskov, R. Bouziane, S. Pachnicke, H. Griesser, B.C. Thomsen, P. Bayvel, and R.I. Killey, "Nyquistshaped dispersion-precompensated subcarrier modulation with direct detection for spectrally-efficient WDM transmission," Opt. Express, vol. 22, no. 8, pp. 9420-9431, 2014.

[9] M.S. Erkılınç, S. Pachnicke, H. Griesser, B.C. Thomsen, P. Bayvel, and R. Killey, "Performance comparison of single sideband direct detection Nyquist-subcarrier modulation and OFDM," in J. Lightw. Technol., vol. 33, no. 10, pp. 2038-2046, 2015. 
[10] M.S. Erkılınç, Z. Li, S. Pachnicke, H. Griesser, B.C. Thomsen, P. Bayvel, and R. Killey, "Spectrally-efficient WDM Nyquist-pulse-shaped 16QAM subcarrier modulation transmission with direct detection," in $J$. Lightw. Technol., vol. 33, no. 15, pp. 3147-3155, 2015.

[11] B.J.C. Schmidt, A.J. Lowery, and J. Armstrong, "Experimental demonstrations of electronic dispersion compensation for long-haul transmission using direct-detection optical OFDM," J. Lightw. Technol., vol. 26, no. 1, pp. 196-203, 2008.

[12] W.R. Peng, B. Zhang, K-.M. Feng, X. Wu, A.E. Willner, and S. Chi, "Spectrally efficient direct-detected OFDM transmission incorporating a tunable frequency gap and an iterative detection techniques," J. Lightw. Technol., vol. 27, no. 24, pp. 5723-5735, 2009.

[13] Y. Zhang, M. O'Sullivan, R. Hui, "Theoretical and experimental investigation of compatible SSB modulation for single channel long-distance optical OFDM transmission," Opt. Express, vol. 18, no. 16, pp. 1675116764, 2010.

[14] V. Vujicic, P.M. Anandarajah, C. Browning, and L.P. Barry, "WDMOFDM-PON based on compatible SSB technique using a mode locked comb source," IEEE Photon. Technol. Lett., vol. 25, no. 21, pp. 20582061, 2013.

[15] M.S. Erkilınç, S. Kilmurray, R. Maher, M. Paskov, R. Bouziane, S. Pachnicke, H. Griesser, B.C. Thomsen, P. Bayvel, and R.I. Killey, "Dispersion-precompensated direct-detection Nyquist-pulse-shaped subcarrier modulation using a dual-drive Mach-Zehnder modulator," to be presented in Proc. OECC, 2015.

[16] M.P. Thakur, M.C.R. Medeiros, P. Laurêncio, and J.E. Mitchell, "Optical frequency tripling with improved suppression and sideband selection," Opt. Express, vol. 19, no. 26, pp. B459-B470, 2011.

[17] A. Dochhan, H. Grieser, M. Eiselt, and J.P. Elbers, "Flexible bandwidth $448 \mathrm{~Gb} / \mathrm{s}$ DMT transmission for next generation data center interConnects," in Proc. ECOC, paper P.4.10, 2014,

[18] R.I. Killey, P.M. Watts, M. Glick, and P. Bayvel, "Electronic dispersion compensation by signal predistortion using digital processing and a dual-drive Mach-Zehnder modulator," IEEE Photon. Technol. Lett., vol.17, no.3, pp.714-716, 2005.

[19] J. McNicol, K. Parsons, L. Strawczynski, and K.B Roberts, "Electrical domain compensation of optical dispersion," Proc. OFC 2005, paper OThJ3, 2005.

[20] D. McGhan, M. O'Sullivan, C. Bontu, and K. Roberts, "Electronic dispersion compensation," Proc. OFC, tutorial, 2006.

[21] M. Seimetz, "High-order modulation for optical fiber transmission," Springer, pp. 18-21, 2009.

[22] K.-P. Ho, and H.-W. Cuei, "Electronic dispersion compensation by signal predistortion using digital processing and a dual-drive Mach-Zehnder modulator," J. of Lightw. Technol., vol. 23, no. 2, pp.764-770, 2005.

[23] D.J.F. Barros and J.M. Kahn, "Optical modulator optimization for orthogonal frequency-division multiplexing," J. of Lightw. Technol., vol. 27, no. 13, pp.2370-2378, 2009.

[24] V. Cizek, "Discrete Hilbert transform," IEEE Trans. Audio Electroacoust., vol. 18, no. 4, pp. 340-343, 1970.

[25] O.O. Omomukuyo, "Orthogonal frequency division multiplexing for optical access networks," Ph.D. dissertation, Dept. Electron. and Elect. Eng., Univ. Coll. London, London, UK, pp. 47-55, 2013.

[26] M. Sieben, J. Conradi, and D.E. Dodds, "Optical single sideband transmission at $10 \mathrm{~Gb} / \mathrm{s}$ using only electrical dispersion compensation," J. Lightw. Technol., vol. 17, no. 10, pp. 1742-1749, 1999.

[27] G.P. Agrawal, "Applications of nonlinear fiber optics," 3rd ed., Academic press, 2010.

[28] C.E. Shannon, "A mathematical theory of communication," Bell Syst. Tech. J., vol. 27, no. 3, pp. 379-423, 1948.

[29] R.A. Shafik, S. Rahman, and A.H.M. Islam, "On the extended relationships among EVM, BER and SNR as performance metrics," in Proc.

ICECE, pp. 408-411, 2006. 